\title{
Wearing Face Masks - the Simple and Effective Way to Block the Infection Source of COVID-19
}

\author{
Tianxing Jiang'; Hao Wang²; George F. Gao ${ }^{3}$; Xiaoming Jiang ${ }^{4,5, \#}$
}

The key difference between the coronavirus disease 2019 (COVID-19) and previous coronaviruses such as SARS and MERS is that some infected people do not display obvious symptoms but are contagious during the incubation period for 14 days (1) or longer.

Recently, there are many discussions about whether all persons should wear masks when they go out during the COVID-19 pandemic. The main reasons for the opposition are that the effectiveness of masks in preventing infection is not clear, ordinary people rarely wear masks correctly, everyone wearing masks may cause social panic, there are not enough masks, etc. (2-3). However, the main reason for supporting wearing a mask is that it can prevent persons with asymptomatic infections (so called covert infections) from releasing virus-containing droplets to infect other people (4-5). George F. Gao of China CDC also pointed out that European and American countries not wearing masks was a mistake and discussed the importance of wearing masks for epidemic control for the first time (4).

This paper is based on the three major measures of prevention and control of infectious diseases and systematic comparisons of the evolutions in practices implemented by epidemic countries with and without mask wearing. We suggest that covert infections actually cause a big leak in controlling the source of infection and inevitably lead to epidemics growing beyond control. "Everyone wear face masks" is a simple, feasible, and low-cost method of blocking the infection source and can result in the epidemic being effectively controlled as evidenced in China and the Republic of Korea.

\section{Leaks in the Control of Infection Source}

Controlling the infection source, stopping the route of transmission, and protecting susceptible people are the three major measures for the prevention and control of infectious diseases. Among them, controlling the infection source successfully is the most important and essential measure since any leaks from the infection source will cause the other two measures to fail.

Specific to the COVID-19 virus epidemic, patients with covert infections are indistinguishable from healthy people without nucleic acid testing. Therefore, patients with covert infections are possibly ignored by the prevention and control departments, the public, and even the infected people themselves, and these patients can become freely moving infection sources, resulting in transmission beyond control. A reported $30 \%-60 \%$ of COVID-19 infections are asymptomatic or have mild symptoms while having the ability to spread the virus $(G)$.

Finding an effective way to distinguish and then control these covert infections is crucial for control of the COVID-19 epidemic. Otherwise, stopping the spread of the virus will be impossible.

\section{Everyone Wear Face Masks: Two Birds, One Stone}

At present, the COVID-19 epidemic are present in many countries in spite of strict quarantine measures, including lockdowns at the city or country level, that have been implemented. Confirmed cases increase rapidly, at times nearly exponentially in some countries, which indicates that the implementation of certain control measures often did not work. China and the Republic of Korea are major exceptions as effective control of the epidemic was achieved within almost a month of the inception, and China had even achieved almost zero growth of local infections (7).

What is the reason for such a big difference in epidemic control? In China and the Republic of Korea, everyone wears face masks.

COVID-19 is mainly transmitted by droplets and close contacts. The virus-bearing droplets spread to the environment while the infected person breathes, talks, and sneezes, and the droplets deposit on the ground, on the table, on the door knob of the bus, and on all objects within a region of about one meter around the 
infected person. All of the deposited droplets become infection sources for several hours, and in many countries' quarantine measures, only infected persons are required to wear masks to prevent them from releasing virus droplets and contain most of the virus in the mask (4-5). However, since persons with asymptomatic covert infections are unknown without nucleic acid testing, these patients then become dangerous sources to spread the COVID-19 virus unintentionally.

In China's and the Republic of Korea's quarantine measures, all people (therefore including the persons with asymptomatic covert infections) are strictly required to wear masks to protect themselves from virus infection. This requirement effectively limits all persons with covert infections from releasing virus droplets so that they cannot become hidden sources of contagion. Two birds, one stone. With everyone wearing face masks, China and the Republic of Korea have used this simple and low cost method to successfully cut off the path of transmission and to block off the invisible infection sources of COVID-19. This latter function is more important and unique for COVID-19 epidemic control.

We can use the epidemics in Guangdong (as a representative of China), the Republic of Korea, and Singapore to illustrate the importance of face masks in epidemic control as these are regions with imported COVID-19 virus only and different measures are being used for epidemic control (7). In Guangdong and the Republic of Korea, the epidemic was effectively controlled with total confirmed cases of less than 1,500 (Guangdong) and about 10,000 (Republic of Korea) within about 10 days of implementing "everyone wear face masks" and other extremely strict measures. Singapore had also adopted strict prevention and control measures and had achieved good results with no serious outbreaks so far. But without the "everyone wear face masks" measure, the country's epidemic control conditions are relatively fragile and seem to be affected more readily by persons with covert infections as local infections take place. The recent continuous increase of infection persons in Singapore may show some instability in their epidemic control strategy and may demonstrate the need for a wider measure involving face masks.

Based on the above discussion and the experiences of the worldwide pandemic, "everyone wear face masks" is playing important roles in cutting off the transmission paths and as the major method of blocking the COVID-19 infection source. Therefore, "everyone wear face masks" is of key importance in epidemic control and is absolutely necessary to fully promote.

Acknowledgements: This work is supported by the "innovation practice training plan for college students of the Chinese Academy of Sciences", and the NSFC under contract U1732108.

\# Corresponding author: Xiaoming Jiang, jiangxm@basic.cas.cn.

${ }^{1}$ China University of Geosciences, Beijing, China; ${ }^{2}$ National Center for Nanoscience and Technology, CAS, Beijing, China; ${ }^{3}$ Chinese Center for Disease Control and Prevention, Beijing, China; ${ }^{4}$ Beijing Advanced Science and Innovation Center, CAS, Beijing, China;

${ }^{5}$ Institute of High Energy Physics, CAS, Beijing, China.

Submitted: April 09, 2020; Accepted: April 09, 2020

\section{REFERENCES}

1. Zhang B. The infectivity of novel coronavirus incubation period should be emphasized. http://www.chinanews.com/sh/2020/01-22/9067646. shtml. [2020-01-22]. (In Chinese).

2. Marasinghe KM. A systematic review investigating the effectiveness of face mask use in limiting the spread of COVID-19 among medically not diagnosed individuals: shedding light on current recommendations provided to individuals not medically diagnosed with COVID-19. Research Square 2020. http://dx.doi.org/10.21203/rs.3.rs-16701/v2. [2020-03-31].

3. Long Y, Hu T, Liu L, Chen R, Guo Q, Yang L, et al. Effectiveness of N95 respirators versus surgical masks against influenza: a systematic review and meta-analysis. J Evid Based Med 2020. http://dx.doi.org/ 10.1111/jebm.12381. [2020-03-13].

4. Cohen J. Not wearing masks to protect against coronavirus is a 'big mistake', top Chinese scientist says. Science 2020. http://dx.doi.org/ 10.1126/science.abb9368. [2020-03-27].

5. Servick K. Would everyone wearing face masks help us slow the pandemic? Science 2020. http://dx.doi.org/10.1126/science.abb9371. [2020-03-28].

6. Qiu J. Covert coronavirus infections could be seeding new outbreaks. Nature 2020. http://dx.doi.org/10.1038/d41586-020-00822-x. [202003-20].

7. WHO. Coronavirus disease (COVID-19) situation dashboard. https:// www.who.int/. [2020-04-9]. 